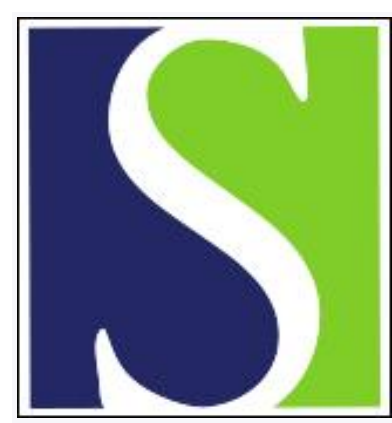

Scand J Work Environ Health 2005;31(4):307-315

https://doi.org/10.5271/sjweh.887

Issue date: Aug 2005

Health effects of cadmium exposure in the general environment in Japan with special reference to the lower limit of the benchmark dose as the threshold level of urinary cadmium

by Uno T, Kobayashi E, Suwazono Y, Okubo Y, Miura K, Sakata K, Okayama A, Ueshima $H$, Nakagawa $H$, Nogawa K

Affiliation: Department of Occupational and Environmental Medicine (A2), Graduate School of Medicine, Chiba University, 1-8-1 Inohana, Chuohku, Chiba, 260-8670 Japan.

Key terms: benchmark dose (lower limit); cadmium exposure; general environment; health effect; Japan; renal effect; threshold level; urinary cadmium

This article in PubMed: www.ncbi.nlm.nih.gov/pubmed/16161714 


\title{
Health effects of cadmium exposure in the general environment in Japan with special reference to the lower limit of the benchmark dose as the threshold level of urinary cadmium
}

\author{
by Tsukasa Uno, MD, ${ }^{1}$ Etsuko Kobayashi, PhD, ${ }^{1}$ Yasushi Suwazono, MD, ${ }^{1}$ Yasushi Okubo, MD, ${ }^{1}$ Katsuyu- \\ ki Miura, MD, ${ }^{2}$ Kiyomi Sakata, MD, ${ }^{3}$ Akira Okayama, MD, ${ }^{4}$ Hirotsugu Ueshima, MD, ${ }^{5}$ Hideaki Nakagawa, \\ $M D,{ }^{2}$ Koji Nogawa, $M D^{1}$
}

Uno T, Kobayashi E, Suwazono Y, Okubo Y, Miura K, Sakata K, Okayama A, Ueshima H, Nakagawa H, Nogawa K. Health effects of cadmium exposure in the general environment in Japan with special reference to the lower limit of the benchmark dose as the threshold level of urinary cadmium. Scand J Work Environ Health 2005;31(4):307-315.

\begin{abstract}
Objectives This study investigates renal dysfunction in areas without known environmental cadmium pollution and calculates the threshold level of urinary cadmium.

Methods Urinary total protein, $\beta_{2}$-microglobulin $\left(\beta_{2}-\mathrm{MG}\right)$, and $\mathrm{N}$-acetyl- $\beta$-D-glucosaminidase (NAG), used as indicators of renal dysfunction, and urinary cadmium concentration, used as an indicator of cadmium exposure, were measured in two sets of 24-hour urine samples from each of 828 participants (410 men, 418 women), aged 40-59 years and living in three areas without any known environmental cadmium pollution. In multiple regression and logistic regression analyses the association between indicators of cadmium exposure and indicators of renal dysfunction were studied. The lower 95\% confidence limit of the dose (benchmark dose) corresponding to a $5 \%\left(\mathrm{BMDL}_{5}\right)$ or $10 \%\left(\mathrm{BMDL}_{10}\right)$ level of each indicator of renal dysfunction above the background level) was calculated as the threshold level of urinary cadmium.

Results With all the expressed units $\left[\mathrm{g}_{\text {creatinine }}{ }^{-1}\right.$ and day $\left.{ }^{-1}\right]$ in the multiple regression analysis, the partial regression coefficients showed a significant association between urinary cadmium concentration and total protein, $\beta_{2}-\mathrm{MG}$, and NAG for both genders, except for total protein for women $\left(\mathrm{g}\right.$ creatinine ${ }^{-1}$ and day $\left.{ }^{-1}\right)$. The same results were obtained for both genders in the logistic regression analysis. The $\mathrm{BMDL}_{10}$ was $0.6-1.2 \mu \mathrm{g} / \mathrm{g}$ creatinine and $0.8-1.6 \mu \mathrm{g} / \mathrm{day}$ for the men and $1.2-3.6 \mu \mathrm{g} / \mathrm{g}$ creatinine, and $0.5-4.7 \mu \mathrm{g} / \mathrm{day}$ for the women.

Conclusions Cadmium exposure and the levels of the indicators of renal dysfunction were associated among the men and women aged 40-59 years in areas without any known environmental cadmium pollution. The threshold level of urinary cadmium in Japan seems to be almost the same as in Belgium and Sweden.
\end{abstract}

Key terms renal effects.

It is well known that long-term exposure to cadmium $(\mathrm{Cd})$ in the general environment causes renal dysfunction $(1,2)$. Over the past several years, we have demonstrated a dose-response relationship between indicators of health effects and the cadmium concentration in rice, lifetime cadmium intake as an indicator of external cadmium exposure, and the cadmium concentration in urine as an indicator of internal dose and have calculated threshold levels of cadmium exposure (3-12). As a result, we found that the tolerable levels of cadmium in rice in cadmium-polluted areas of the Kakehashi River basin in the Ishikawa Prefecture and the Jinzu River basin in the Toyama Prefecture were lower than 0.4 ppm, the level over which the Japanese government prohibits trading (3-5). We have reported that the tolerable level of lifetime cadmium intake for both genders in the Kakehashi River basin is about 2 grams (6-8), and less than 1.58 grams in the Jinzu River basin (12). These levels of lifetime cadmium intake in both areas were small, being equal to or less than twofold the

1 Department of Occupational and Environmental Medicine, Graduate School of Medicine, Chiba University, Chiba, Japan.

2 Department of Public Health, Kanazawa Medical University, Kanazawa, Japan.

3 Department of Public Health, Wakayama Medical University, Wakayama, Japan.

4 Department of Preventive Cardiology, National Cardiovascular Center, Osaka, Japan.

5 Department of Health Science, Shiga University of Medical Science, Otsu, Japan.

Reprint requests to: Dr Etsuko Kobayashi, Department of Occupational and Environmental Medicine (A2), Graduate School of Medicine, Chiba University, 1-8-1 Inohana, Chuohku, Chiba, 260-8670 Japan. [E-mail: ekoba@ faculty.chiba-u.jp] 
quantity of cadmium intake in the general environment in Japan. For inhabitants of cadmium-polluted areas in the Kakehashi River basin, the threshold level of urinary cadmium calculated from our previous study was estimated to be 1.6-3.0 $\mu \mathrm{g} / \mathrm{g}$ creatinine (cr) for men and $2.3-4.6 \mu \mathrm{g} / \mathrm{g} \mathrm{cr}$ for women; these values were almost the same as the mean urinary cadmium concentration in the control group (11). In other words, it became clear that the margin between the threshold level of cadmium exposure and the levels of cadmium exposure in the control group was not big even when the calculation was done using different indicators of cadmium exposure. When these results are considered, it is possible that renal effects are induced by exposure to cadmium even in a so-called cadmium unpolluted area of the general environment in Japan. Thus we next investigated the association between the cadmium concentration in either blood or urine and indicators of renal dysfunction, including total protein, $\beta_{2}$-microglobulin $\left(\beta_{2}-\mathrm{MG}\right)$, and $\mathrm{N}$ acetyl- $\beta$-D-glucosaminidase (NAG) for a total of 2753 inhabitants $>50$ years of age (1105 men, 1648 women) in three areas not polluted by cadmium. As a result, we showed the presence of renal effects induced by cadmium exposure in the general environment without any known cadmium pollution (13-15). In contrast, Ikeda and his colleagues carried out a similar study, using blood and urine samples collected from 607 women who lived in areas of 30 survey sites in prefectures throughout Japan, but they could not find a clear dose-response relationship between cadmium exposure and indicators of renal effects (16). Therefore, in this study, we investigated the presence of renal effects induced by cadmium exposure using 24-hour urine samples of men and women aged 40-59 years in three areas different from those in the previous studies. Furthermore, the lower limit of a benchmark dose (BMDL = benchmark dose, low) was calculated from a benchmark dose (BMD) as the threshold level for urinary cadmium.

\section{Study population and methods}

\section{Study population}

The INTERMAP study (17), a basic epidemiologic investigation, was performed to clarify unanswered questions on the role of multiple dietary factors in the etiology of unfavorable blood pressure patterns that prevail in most middle-aged and older people. It is an international cooperative 17-sample population study of 4660 men and women aged 40-59 years, conducted in four countries (China, Japan, the United Kingdom, the United States). The 24-hour urine collections were obtained twice per participant in the study (18). We obtained the 24-urine specimens from three centers in Japan (Toyama,
Shiga, and Wakayama) in 1997 and 1998. Participants were selected randomly from population lists and stratified by age and gender to give approximately equal numbers (65 persons) in each of two 10-year age and gender groups. The participation rate among the selected participants was $93 \%$ in Japan (17). The target group comprised 828 participants (410 men, 418 women, who collected urine samples both times). It consisted of 292 participants from Toyama (145 men, 147 women), 249 participants from Shiga (121 men, 128 women), and 287 participants from Wakayama (144 men, 143 women). No participant in the target population had occupational exposure to heavy metals. Smokers and nonsmokers were included among the participants examined since smoking habit did not significantly affect the relationship between cadmium exposure and renal effects in the previous study on the general environment in Japan (15).

\section{Collection of samples and analytical method}

In this study, we obtained two sets of 24-hour urine samples from each participant and decided to use the mean. The two values of excreted substances measured in the 24-hour urine samples obtained from the same participant agreed well [creatinine: $r=0.82$, coefficient of variation $(\mathrm{CV})=31.2$; protein: $\mathrm{r}=0.87, \mathrm{CV}=143.5 ; \beta_{2^{-}}$ MG: $r=0.71, C V=140.5$; NAG: $r=0.54, C V=102.5$; $\mathrm{Cd}: \mathrm{r}=0.94, \mathrm{CV}=97.5]$. Thus we thought that the excretion levels of substances in the 24-hour urine samples were stable.

The 24-hour urine samples consisted of samples collected every 4 hours as one unit and put into six polyethylene containers. Boric acid was added to the polyethylene containers beforehand as an antiseptic. The participants recorded the beginning and ending of the sampling time, and we corrected it precisely to 24 hours. At the end of the sampling, the participants were asked to describe the conditions of the urine sampling by selecting one of the following: (i) spilled several drops from the container, (ii) spilled more than several drops, (iii) saved all the urine. The urine samples from which more than several drops were spilled were excluded from this analysis. The total urine specimens were mixed well and put into polyethylene bottles. Thereafter the polyethylene bottles were kept frozen at $-20^{\circ} \mathrm{C}$ until the analysis. The containers and polyethylene bottles were checked for cadmium, and cadmium was not detected. All of the urinary samples were $>$ pH 5.5 since boric acid was added to the polyethylene container beforehand. The urinary indicators of renal dysfunction were the same as those used in previous studies (ie, total protein, $\beta_{2}-\mathrm{MG}$, and NAG) (13-15). Total protein was analyzed using a kit (Tonein-TP, Otsuka Pharmacy, Japan), $\beta_{2}-\mathrm{MG}$ was measured for all specimens by radioimmunoassay (Pharmacia $\beta_{2}$-micro RIA, Pharmacia Diag- 
nostics AB, Sweden), and NAG was analyzed using a kit (NAG test Shionogi, Shionogi Pharmaceuticals, Japan). The urinary cadmium concentration was measured by graphite-furnace atomic absorption spectrometry using a Hitachi Model Z-8100 (19). Creatinine was determined by the Jaffe reaction method (20). The detection limits were $1 \mathrm{mg} / \mathrm{l}$ for protein, $1 \mu \mathrm{g} / \mathrm{l}$ for $\beta_{2}-\mathrm{MG}, 0.05 \mathrm{U} / \mathrm{l}$ for $\mathrm{NAG}, 0.05 \mu \mathrm{g} / \mathrm{l}$ for cadmium, and $0.005 \mathrm{~g} / \mathrm{l}$ for creatinine. The degree of imprecision of each analysis was estimated from duplicate measurements. The correlation coefficients among the double samples were $0.98-1.00$ and the slopes of all the correlation equations were about 1.0. The accuracy of the measurements was checked using control urine or serum (observed value/the standard one $=96-102$ ).

\section{Statistical analysis}

The values were expressed in the following two ways: corrected creatinine unit $\left(\mathrm{g} \mathrm{cr}^{-1}\right)$ and 24-hour excretion $\left(\right.$ day $\left.^{-1}\right)$. In the multiple regression model, total protein, $\beta_{2}-\mathrm{MG}$, and NAG, as indicators of renal dysfunction, were used as criterion variables, age and urinary cadmium excretion (an indicator of cadmium exposure) were the explanatory variables, and the analysis was performed for the men and women separately. In the logistic model, total protein, $\beta_{2}-\mathrm{MG}$, and NAG were employed as criterion variables, age and urinary cadmium excretion were the explanatory variables (continuous variables), and the analysis was performed for the men and women separately. The cut-off values for the criterion variables were defined as the $84 \%$ upper limit values (geometric mean $\times$ geometric standard deviation), which were calculated from the values of the target population on the assumption of a log-normal distribution. The cut-off values of each substance are shown in table 1 for each expressed unit and gender. Urinary cadmium excretion was divided into four to six subgroups by each expressed unit, and the prevalence of each urinary substance in each subgroup was calcu- lated using the $84 \%$ upper limit value of this group. Then we calculated the BMD and the BMDL. BMD 10 or $\mathrm{BMD}_{5}$ is the cadmium excretion level in urine that can be expected to yield an excess prevalence of abnormal levels of the substance used as an indicator for renal dysfunction of 0.10 or 0.05 , respectively. BMDL is the cadmium excretion of the lower limit in urine of the $95 \%$ confidence intervals of the $\operatorname{BMD}(21,22)$. The analysis of the regression and curve estimation was performed with Benchmark Dose Software (version 3.1.1) available from the United States Environmental Protection Agency (EPA). The BMD and BMDL values were calculated by a quantal linear model when a distribution of the prevalence of abnormal levels of the substance used as the indicator for renal dysfunction fit their model. A significant difference was found between the expected values and the observed ones in the case of a quantal linear model for NAG $\left(\right.$ day $\left.^{-1}\right)$ for the women $(\mathrm{P}=0.03)$. Thus a log-logistic model was instead fit to the NAG $\left(\right.$ day $\left.^{-1}\right)$ for the women.

\section{Results}

The number of participants examined, the median, and the $5 \%$ and $95 \%$ values for age, the volume of urine, and the excretion of creatinine, cadmium, total protein, $\beta_{2}-\mathrm{MG}$, and NAG are shown in table 2 according to the area of residence and gender.

Table 1. Cut-off values a for total protein, $\beta_{2}$-microglobulin $\left(\beta_{2}{ }^{-}\right.$ $M G)$, and $N$-acetyl- $\beta$-D-glucosaminidase (NAG) in urine. $(\mathrm{cr}=$ creatinine)

\begin{tabular}{|c|c|c|c|c|c|c|}
\hline \multirow[t]{2}{*}{ Gender } & \multicolumn{2}{|c|}{ Total protein } & \multicolumn{2}{|c|}{$\beta_{2}-M G$} & \multicolumn{2}{|c|}{ NAG } \\
\hline & $\mathrm{mg} / \mathrm{g} \mathrm{cr}$ & $\mathrm{mg} / \mathrm{day}$ & $\mu \mathrm{g} / \mathrm{g} \mathrm{cr}$ & $\mu \mathrm{g} /$ day & $\mathrm{U} / \mathrm{g} \mathrm{cr}$ & U/day \\
\hline Male & 70 & 104 & 233 & 340 & 2.4 & 3.4 \\
\hline Female & 79 & 75 & 274 & 262 & 2.5 & 2.4 \\
\hline
\end{tabular}

a The $84 \%$ upper limit values (geometric mean $\times$ geometric standard deviation), calculated from the values of the target population on the assumption of a log-normal distribution.

Table 2. Urinalysis by area. $(\mathrm{cr}=$ creatinine, $\mathrm{M}=$ median, $5=5 \%$ percentile, $95=95 \%$ percentile, $\mathrm{TP}=$ Toyama prefecture, $\mathrm{SP}=\mathrm{Shiga}$ prefecture, WP = Wakayama prefecture)

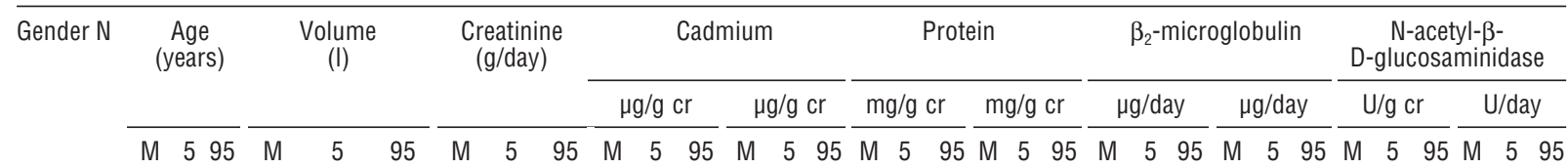

Men

TP $\quad 145 \quad 5042571.4860 .9712 .2691 .421 .031 .812 .0 \quad 0.7 \quad 4.52 .8 \quad 0.96 .84525142 \quad 62 \quad 38 \quad 161155 \quad 66449214896241.7 \quad 0.4 \quad 4.92 .5 \quad 0.6 \quad 6.5$

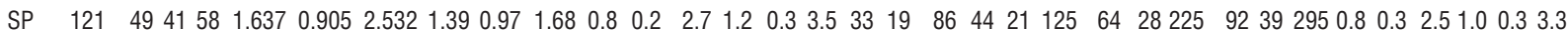

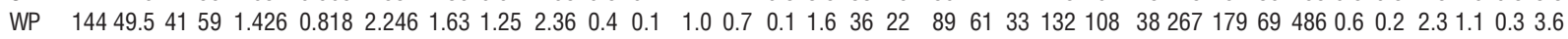

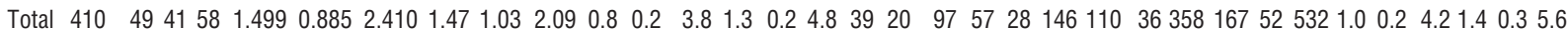
Women

TP $\quad 147 \quad 4941571.3030 .8402 .0510 .93 \quad 0.691 .204 .2 \quad 1.910 .1 \quad 3.91 .79 .753 \quad 3611548 \quad 33107200101468172964462.1 \quad 0.74 .31 .8 \quad 0.74 .3$

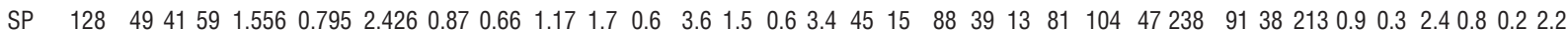

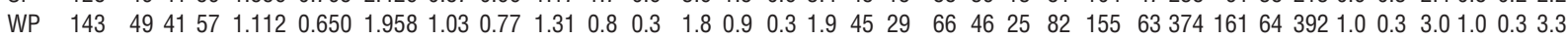

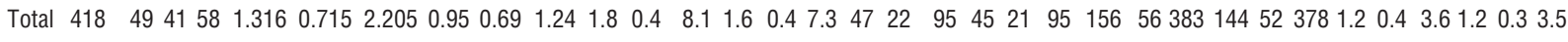


Table 3. Median, $5 \%$ percentile ( $5 \%$ ) and $95 \%$ percentile (95\%) of protein, $\beta_{2}$-microglobulin ( $\beta_{2}-M G$ ), and $N$-acetyl- $\beta$-D-glucosaminidase (NAG) in urine by gender and urinary cadmium levels. ( $N=$ number of subjects examined)

\begin{tabular}{|c|c|c|c|c|c|c|c|c|c|c|c|c|}
\hline \multirow[t]{2}{*}{ Cadmium level } & \multicolumn{4}{|c|}{ Protein (mg/g cr) } & \multicolumn{4}{|c|}{$\beta_{2}-\mathrm{MG}(\mu \mathrm{g} / \mathrm{g} \mathrm{cr})$} & \multicolumn{4}{|c|}{ NAG (U/g cr) } \\
\hline & $\mathrm{N}$ & Median & $5 \%$ & $95 \%$ & $\mathrm{~N}$ & Median & $5 \%$ & $95 \%$ & $\mathrm{~N}$ & Median & $5 \%$ & $95 \%$ \\
\hline \multicolumn{13}{|l|}{$\leq 0.9 \mu \mathrm{g} / \mathrm{g} \mathrm{cr}$} \\
\hline Men & 229 & 35 & 19 & 86 & 229 & 95 & 35 & 234 & 229 & 0.7 & 0.2 & 2.1 \\
\hline Women & 116 & 44 & 21 & 118 & 116 & 137 & 57 & 417 & 116 & 0.9 & 0.2 & 2.9 \\
\hline \multicolumn{13}{|l|}{$1.0-1.9 \mu \mathrm{g} / \mathrm{g} \mathrm{cr}$} \\
\hline Men & 97 & 40 & 18 & 97 & 97 & 121 & 39 & 449 & 97 & 1.1 & 0.2 & 4.2 \\
\hline Women & 120 & 45 & 19 & 77 & 120 & 128 & 48 & 287 & 120 & 0.9 & 0.3 & 3.0 \\
\hline \multicolumn{13}{|l|}{$2.0-2.9 \mu \mathrm{g} / \mathrm{g} \mathrm{cr}$} \\
\hline Men & 45 & 45 & 24 & 83 & 45 & 150 & 47 & 683 & 45 & 1.9 & 0.5 & 6.1 \\
\hline Women & 59 & 54 & 32 & 94 & 59 & 167 & 64 & 342 & 59 & 1.4 & 0.4 & 3.9 \\
\hline \multicolumn{13}{|l|}{$3.0-3.9 \mu \mathrm{g} / \mathrm{g} \mathrm{cr}$} \\
\hline Men & 24 & 47 & 25 & 82 & 24 & 185 & 21 & 417 & 24 & 2.2 & 0.2 & 8.3 \\
\hline Women & 38 & 50 & 23 & 82 & 38 & 180 & 58 & 469 & 38 & 1.5 & 0.4 & 4.5 \\
\hline \multicolumn{13}{|l|}{$4.0-4.9 \mu \mathrm{g} / \mathrm{g} \mathrm{cr}$} \\
\hline Men & 10 & 60 & 45 & 241 & 10 & 248 & 80 & 1263 & 10 & 3.1 & 0.7 & 6.7 \\
\hline Women & 29 & 50 & 31 & 113 & 29 & 199 & 108 & 353 & 29 & 1.7 & 0.4 & 3.7 \\
\hline \multicolumn{13}{|l|}{$5.0-9.9 \mu \mathrm{g} / \mathrm{g} \mathrm{cr}$} \\
\hline Men & 5 & 45 & 36 & 287 & 5 & 145 & 102 & 1579 & 5 & 1.2 & 0.9 & 2.0 \\
\hline Women & 48 & 54 & 24 & 118 & 48 & 197 & 77 & 483 & 48 & 2.1 & 0.7 & 4.3 \\
\hline \multicolumn{13}{|l|}{$\geq 10.0 \mu \mathrm{g} / \mathrm{g} \mathrm{cr}$} \\
\hline Men & 0 & . & . & . & 0 & . & . & . & 0 & . & . & . \\
\hline Women & 8 & 67 & 46 & 129 & 8 & 346 & 207 & 2144 & 8 & 2.8 & 1.4 & 7.5 \\
\hline \multicolumn{13}{|l|}{ Total } \\
\hline Men & 410 & 39 & 20 & 97 & 410 & 110 & 36 & 358 & 410 & 1.0 & 0.2 & 4.2 \\
\hline Women & 418 & 47 & 23 & 94 & 418 & 156 & 56 & 374 & 418 & 1.2 & 0.3 & 3.5 \\
\hline
\end{tabular}

Table 4. Median, $5 \%$ and $95 \%$ percentile of protein, $\beta_{2}$-microglobulin ( $\beta_{2}-M G$ ), and $N$-acetyl- $\beta$-D-glucosaminidase (NAG) in urine by gender and urinary cadmium levels.

\begin{tabular}{|c|c|c|c|c|c|c|c|c|c|c|c|c|}
\hline \multirow[t]{2}{*}{ Cadmium level } & \multicolumn{4}{|c|}{ Protein (mg/day) } & \multicolumn{4}{|c|}{$\beta_{2}-M G(\mu \mathrm{g} /$ day $)$} & \multicolumn{4}{|c|}{ NAG (U/day) } \\
\hline & $\mathrm{N}$ & Median & $5 \%$ & $95 \%$ & $\mathrm{~N}$ & Median & $5 \%$ & $95 \%$ & $\mathrm{~N}$ & Median & $5 \%$ & $95 \%$ \\
\hline \multicolumn{13}{|l|}{$\leq 0.9 \mu \mathrm{g} /$ day } \\
\hline Men & 159 & 54 & 26 & 105 & 159 & 150 & 51 & 344 & 159 & 1.0 & 0.3 & 3.2 \\
\hline Women & 123 & 45 & 18 & 84 & 123 & 138 & 48 & 379 & 123 & 0.8 & 0.2 & 2.9 \\
\hline \multicolumn{13}{|l|}{$1.0-1.9 \mu \mathrm{g} /$ day } \\
\hline Men & 122 & 56 & 26 & 125 & 122 & 152 & 47 & 365 & 122 & 1.3 & 0.4 & 4.4 \\
\hline Women & 123 & 43 & 17 & 68 & 123 & 112 & 45 & 242 & 123 & 1.0 & 0.2 & 2.4 \\
\hline \multicolumn{13}{|l|}{$2.0-2.9 \mu \mathrm{g} /$ day } \\
\hline Men & 52 & 63 & 33 & 132 & 52 & 223 & 80 & 578 & 52 & 2.2 & 0.5 & 6.0 \\
\hline Women & 62 & 46 & 21 & 70 & 62 & 148 & 47 & 305 & 62 & 1.4 & 0.3 & 3.8 \\
\hline \multicolumn{13}{|l|}{$3.0-3.9 \mu \mathrm{g} /$ day } \\
\hline Men & 36 & 60 & 39 & 146 & 36 & 192 & 61 & 378 & 36 & 2.5 & 0.5 & 5.3 \\
\hline Women & 37 & 47 & 28 & 72 & 37 & 188 & 85 & 374 & 37 & 1.5 & 0.5 & 3.5 \\
\hline \multicolumn{13}{|l|}{$4.0-4.9 \mu \mathrm{g} /$ day } \\
\hline Men & 24 & 67 & 29 & 255 & 24 & 317 & 23 & 617 & 24 & 4.1 & 0.7 & 8.1 \\
\hline Women & 29 & 48 & 18 & 64 & 29 & 185 & 81 & 267 & 29 & 1.6 & 0.5 & 2.8 \\
\hline \multicolumn{13}{|l|}{$5.0-5.9 \mu \mathrm{g} /$ day } \\
\hline Men & 7 & 70 & 37 & 74 & 7 & 214 & 118 & 312 & 7 & 4.0 & 2.4 & 5.8 \\
\hline Women & 15 & 46 & 26 & 70 & 15 & 160 & 92 & 319 & 15 & 1.5 & 0.3 & 2.8 \\
\hline \multicolumn{13}{|l|}{$\geq 6.0 \mu \mathrm{g} /$ day } \\
\hline Men & 10 & 76 & 41 & 114 & 10 & 212 & 134 & 1865 & 10 & 2.4 & 1.2 & 4.4 \\
\hline Women & 29 & 52 & 21 & 107 & 29 & 247 & 58 & 540 & 29 & 2.0 & 0.9 & 5.1 \\
\hline \multicolumn{13}{|l|}{ Total } \\
\hline Men & 410 & 57 & 28 & 146 & 410 & 167 & 53 & 532 & 410 & 1.4 & 0.3 & 5.6 \\
\hline Women & 418 & 45 & 21 & 94 & 418 & 145 & 52 & 374 & 418 & 1.2 & 0.3 & 3.5 \\
\hline
\end{tabular}


The number of participants examined, the median, and the $5 \%$ and $95 \%$ values for total protein, $\beta_{2}-\mathrm{MG}$, and NAG are shown in table 3 (see p 310) (corrected creatinine unit) and table 4 (see p 310) (24-hour excretion) according to gender and urinary cadmium concentration.

\section{Multiple regression analysis}

The results of the multiple regression analysis are shown in table 5. All of the partial regression coefficients showed a significant association between the urinary cadmium excretion and the total protein, $\beta_{2}-\mathrm{MG}$, and

Table 5. Criterion variables, explanatory variables, partial regression coefficients [ $95 \%$ confidence intervals $(95 \% \mathrm{CI})$ ], and multiple correlation coefficients in the multiple regression analysis of the results from 410 male and 418 female participants. $\left(\beta_{2}-\mathrm{MG}=\right.$ $\beta_{2}$-microglobulin, NAG = N-acetyl- $\beta$-D-glucosaminidase, $\mathrm{U}-\mathrm{Cd}=$ urinary cadmium)

\begin{tabular}{|c|c|c|c|c|}
\hline $\begin{array}{l}\text { Criterion } \\
\text { variable }\end{array}$ & $\begin{array}{l}\text { Partial } \\
\text { regression } \\
\text { coefficient }\end{array}$ & $95 \% \mathrm{Cl}$ & $\begin{array}{l}\text { Multiple } \\
\text { correlation } \\
\text { coefficient }\end{array}$ & P-value \\
\hline \multicolumn{5}{|c|}{ Men $(N=410)$} \\
\hline \multicolumn{5}{|c|}{ Protein[expressed unit: $g$ creatinine ${ }^{-1}$ ] } \\
\hline $\begin{array}{l}\text { Age } \\
\text { U-Cd }\end{array}$ & $\begin{array}{r}0.90 \\
14\end{array}$ & $\begin{array}{c}-0.78-2.6 \\
6.0-21\end{array}$ & 0.17 & $P<0.001$ \\
\hline \multicolumn{5}{|l|}{$\beta_{2}-M G$} \\
\hline Age & 0.83 & $-3.1-4.8$ & & \\
\hline U-Cd & 42 & $24-59$ & 0.22 & $P<0.001$ \\
\hline \multicolumn{5}{|l|}{$N A G$} \\
\hline Age & 0.013 & $-0.011-0.036$ & & \\
\hline U-Cd & 0.51 & $0.40-0.61$ & 0.44 & $P<0.001$ \\
\hline \multicolumn{5}{|c|}{ Protein [expressed unit: day ${ }^{-1}$ ] } \\
\hline Age & 0.71 & $-1.5-2.9$ & & \\
\hline U-Cd & 9.3 & $2.2-17$ & 0.10 & $P=0.02$ \\
\hline \multicolumn{5}{|l|}{$\beta_{2}-M G$} \\
\hline Age & -0.25 & $-6.4-5.9$ & & \\
\hline $\mathrm{U}-\mathrm{Cd}$ & 41 & $21-61$ & 0.17 & $P<0.001$ \\
\hline \multicolumn{5}{|l|}{ NAG } \\
\hline Age & 0.093 & $-0.023-0.042$ & & \\
\hline $\mathrm{U}-\mathrm{Cd}$ & 0.46 & $0.35-0.56$ & 0.39 & $P<0.001$ \\
\hline \multicolumn{5}{|c|}{ Women(N=418) } \\
\hline \multicolumn{5}{|c|}{ Protein[expressed unit: g creatinine-1] } \\
\hline Age & 0.26 & $-0.73-1.3$ & & \\
\hline $\mathrm{U}-\mathrm{Cd}$ & 1.5 & $-0.51-3.4$ & 0.00 & $P=0.23$ \\
\hline \multicolumn{5}{|l|}{$\beta_{2}-M G$} \\
\hline Age & -1.7 & $-4.6-1.3$ & & \\
\hline $\mathrm{U}-\mathrm{Cd}$ & 25 & $19-31$ & 0.38 & $P<0.001$ \\
\hline \multicolumn{5}{|l|}{ NAG } \\
\hline Age & 0.018 & $0.000-0.035$ & & \\
\hline U-Cd & 0.18 & $0.15-0.22$ & 0.47 & $P<0.001$ \\
\hline \multicolumn{5}{|c|}{ Protein [expressed unit: day ${ }^{-1}$ ] } \\
\hline Age & -0.069 & $-0.98-0.85$ & & \\
\hline $\mathrm{U}-\mathrm{Cd}$ & 0.96 & $-1.2-3.1$ & 0.00 & $P>0.30$ \\
\hline \multicolumn{5}{|l|}{$\beta_{2}-M G$} \\
\hline Age & -2.2 & $-4.9-0.48$ & & \\
\hline U-Cd & 19 & $13-26$ & 0.27 & $P<0.001$ \\
\hline \multicolumn{5}{|l|}{ NAG } \\
\hline Age & 0.011 & $-0.0070-0.028$ & & \\
\hline U-Cd & 0.17 & $0.13-0.21$ & 0.38 & $P<0.001$ \\
\hline
\end{tabular}

NAG concentrations, except for the total protein concentration of the women (corrected creatinine unit and 24-h excretion). For the partial regression coefficients, only a corrected unit of NAG for the women was significant with respect to age. All the multiple correlation coefficients were significant, except for the total protein (corrected creatinine unit and 24-h excretion) concentration of the women. The highest multiple correlation coefficients for NAG were 0.44 for the men and 0.47 for the women as corrected for creatinine.

\section{Logistic regression analysis}

The results calculated from the logistic regression model are shown in table 6 . The odds ratios for the urinary cadmium concentration were significantly higher than 1 for

Table 6. Criterion variables, explanatory variables, and odds ratios $[95 \%$ confidence intervals $(95 \% \mathrm{CI})]$ in the logistic regression analysis of the results from the 410 male and 418 female participants. (U-Cd = urinary cadmium)

\begin{tabular}{|c|c|c|}
\hline Criterion variable & Odds ratio & $95 \% \mathrm{Cl}$ \\
\hline \multicolumn{3}{|l|}{ Men } \\
\hline \multicolumn{3}{|c|}{ Protein [expressed unit: $g$ creatinine $\left.{ }^{-1}\right]^{a}$} \\
\hline Age & 1.00 & $0.94-1.01$ \\
\hline U-Cd & 1.45 & $1.15-1.81$ \\
\hline \multicolumn{3}{|c|}{$\beta_{2}-\mathrm{MG}$ [expressed unit: $\mathrm{g}$ creatinine $\left.{ }^{-1}\right]^{\mathrm{a}}$} \\
\hline Age & 1.04 & $0.98-1.11$ \\
\hline U-Cd & 1.71 & $1.38-2.14$ \\
\hline \multicolumn{3}{|c|}{ NAG [expressed unit: $g$ creatinine $\left.e^{-1}\right]^{a}$} \\
\hline Age & 0.99 & $0.94-1.05$ \\
\hline U-Cd & 2.08 & $1.65-2.62$ \\
\hline \multicolumn{3}{|c|}{ Protein [expressed unit: day-1 ${ }^{-1}{ }^{\mathrm{a}}$} \\
\hline Age & 0.98 & $0.92-1.04$ \\
\hline U-Cd & 1.31 & $1.11-1.54$ \\
\hline \multicolumn{3}{|c|}{$\beta_{2}-M G$ [expressed unit: day-1] a $^{a}$} \\
\hline Age & 1.01 & $0.95-1.07$ \\
\hline $\mathrm{U}-\mathrm{Cd}$ & 1.38 & $1.18-.62$ \\
\hline \multicolumn{3}{|c|}{ NAG [expressed unit: day-1 $]^{a}$} \\
\hline Age & 0.99 & $0.94-1.05$ \\
\hline $\mathrm{U}-\mathrm{Cd}$ & 1.60 & $1.36-1.89$ \\
\hline \multicolumn{3}{|l|}{ Women } \\
\hline \multicolumn{3}{|c|}{ Protein [expressed unit: $\mathrm{g}$ creatinine-1 ${ }^{\mathrm{a}}$} \\
\hline Age & 1.01 & $0.93-1.08$ \\
\hline $\mathrm{U}-\mathrm{Cd}$ & 1.16 & $1.05-1.29$ \\
\hline \multicolumn{3}{|c|}{$\beta_{2}-M G$ [expressed unit: $g$ creatinine $\left.{ }^{-1}\right]^{a}$} \\
\hline Age & 1.00 & $0.94-1.06$ \\
\hline U-Cd & 1.35 & $1.22-1.50$ \\
\hline \multicolumn{3}{|c|}{ NAG [expressed unit: $g$ creatinine $\left.{ }^{-1}\right]^{a}$} \\
\hline Age & 1.04 & $0.98-1.10$ \\
\hline U-Cd & 1.25 & $1.13-1.37$ \\
\hline \multicolumn{3}{|c|}{ Protein [expressed unit: day-1 ${ }^{\text {a }}$} \\
\hline Age & 0.96 & $0.89-1.03$ \\
\hline $\mathrm{U}-\mathrm{Cd}$ & 1.18 & $1.04-1.34$ \\
\hline \multicolumn{3}{|c|}{$\beta_{2}-M G$ [expressed unit: day $\left.{ }^{-1}\right]^{a}$} \\
\hline Age & 0.97 & $0.91-1.03$ \\
\hline $\mathrm{U}-\mathrm{Cd}$ & 1.36 & $1.22-1.51$ \\
\hline \multicolumn{3}{|c|}{ NAG [expressed unit: day-1] a } \\
\hline Age & 1.01 & $0.96-1.07$ \\
\hline U-Cd & 1.23 & $1.12-1.37$ \\
\hline
\end{tabular}

a Explanatory variables were used as continuous variables. 
the total protein, $\beta_{2}-\mathrm{MG}$, and NAG concentrations for all the units for both genders. An odds ratio of 1.45 for the men in association with the urinary cadmium (corrected creatinine), as reported in table 6 , implies that the prevalence odds ratio for the abnormal protein level can be expected to increase by a factor 1.45 for each unit of urinary cadmium above zero. The odds ratios for age were not significant for either gender.

\section{Benchmark dose value}

The results of the BMD and BMDL calculated from the quantal linear model (setting an abnormal value above background at $10 \%$ or $5 \%$ ) are shown in table 7 . In addition, the analytical result from the log-logistic model was added, because the abnormal rate distribution of NAG (24-hour excretion) for the women did not fit the quantal linear model. The $\mathrm{BMDL}_{10}$ (setting an abnormal value above background at $10 \%$ ) of the urinary cadmium concentration for the total protein was $1.2 \mu \mathrm{g} / \mathrm{g} \mathrm{cr}$

Table 7. Benchmark dose low (BMDL) estimates of urinary cadmium for proteinuria, $\beta_{2}-M G$-uria, and NAG-uria using quantal linear model. a $\left(B M D=\right.$ benchmark dose, $\beta_{2}-M G=\beta_{2}-$ microglobulin, NAG = N-acetyl- $\beta$-D-glucosaminidase, $\mathrm{cr}=$ creatinine)

\begin{tabular}{|c|c|c|c|c|c|c|c|}
\hline Gender & $\begin{array}{l}\text { Back- } \\
\text { ground }\end{array}$ & Slope & P-value & $\mathrm{BMD}_{10}{ }^{\mathrm{b}}$ & $\mathrm{BMDL}_{10}{ }^{\mathrm{C}}$ & $\mathrm{BMD}_{5}{ }^{\mathrm{d}}$ & $\mathrm{BMDL}_{5}$ \\
\hline \multicolumn{8}{|l|}{ Men } \\
\hline \multicolumn{8}{|l|}{ Protein } \\
\hline $\begin{array}{l}\mathrm{g} \mathrm{cr}^{-1} \\
\text { day }^{-1}\end{array}$ & $\begin{array}{l}0.034 \\
0.044\end{array}$ & $\begin{array}{l}0.059 \\
0.041\end{array}$ & $\begin{array}{l}0.77 \\
0.73\end{array}$ & $\begin{array}{l}1.9 \\
2.7\end{array}$ & $\begin{array}{l}1.2 \\
1.6\end{array}$ & $\begin{array}{l}0.9 \\
1.3\end{array}$ & $\begin{array}{l}0.6 \\
0.8\end{array}$ \\
\hline \multicolumn{8}{|c|}{$\beta_{2}-M G$} \\
\hline $\begin{array}{l}\mathrm{g} \mathrm{cr}^{-1} \\
\text { day }^{-1}\end{array}$ & $\begin{array}{l}0.015 \\
0.024\end{array}$ & $\begin{array}{l}0.107 \\
0.067\end{array}$ & $\begin{array}{l}0.53 \\
0.50\end{array}$ & $\begin{array}{l}1.0 \\
1.6\end{array}$ & $\begin{array}{l}0.7 \\
1.1\end{array}$ & $\begin{array}{l}0.5 \\
0.8\end{array}$ & $\begin{array}{l}0.4 \\
0.5\end{array}$ \\
\hline \multicolumn{8}{|c|}{ NAG } \\
\hline $\begin{array}{l}\mathrm{g} \mathrm{cr}^{-1} \\
\text { day }^{-1}\end{array}$ & $\begin{array}{l}0.000 \\
0.000\end{array}$ & $\begin{array}{l}0.150 \\
0.104\end{array}$ & $\begin{array}{l}0.23 \\
0.42\end{array}$ & $\begin{array}{l}0.7 \\
1.0\end{array}$ & $\begin{array}{l}0.6 \\
0.8\end{array}$ & $\begin{array}{l}0.3 \\
0.5\end{array}$ & $\begin{array}{l}0.3 \\
0.4\end{array}$ \\
\hline \multicolumn{8}{|c|}{ Women } \\
\hline \multicolumn{8}{|c|}{ Protein } \\
\hline $\begin{array}{l}\mathrm{g} \mathrm{cr}^{-1} \\
\text { day }^{-1}\end{array}$ & $\begin{array}{l}0.036 \\
0.053\end{array}$ & $\begin{array}{l}0.016 \\
0.011\end{array}$ & $\begin{array}{l}0.41 \\
0.07\end{array}$ & $\begin{array}{c}6.6 \\
10.3\end{array}$ & $\begin{array}{l}3.6 \\
4.7\end{array}$ & $\begin{array}{l}3.2 \\
5.0\end{array}$ & $\begin{array}{l}1.8 \\
2.3\end{array}$ \\
\hline \multicolumn{8}{|c|}{$\beta_{2}-\mathrm{MG}$} \\
\hline $\begin{array}{l}\mathrm{g} \mathrm{cr}^{-1} \\
\text { day }^{-1}\end{array}$ & $\begin{array}{l}0.015 \\
0.049\end{array}$ & $\begin{array}{l}0.059 \\
0.047\end{array}$ & $\begin{array}{l}0.23 \\
0.06\end{array}$ & $\begin{array}{l}1.8 \\
2.4\end{array}$ & $\begin{array}{l}1.3 \\
1.6\end{array}$ & $\begin{array}{l}0.9 \\
1.2\end{array}$ & $\begin{array}{l}0.7 \\
0.8\end{array}$ \\
\hline \multicolumn{8}{|c|}{$N A G$} \\
\hline $\begin{array}{l}\mathrm{g} \mathrm{cr}^{-1} \\
\text { day }^{-1}\end{array}$ & $\begin{array}{l}0.011 \\
0.000\end{array}$ & $\begin{array}{l}0.068 \\
0.707\end{array}$ & $\begin{array}{l}0.37 \\
0.07\end{array}$ & $\begin{array}{l}1.6 \\
1.0\end{array}$ & $\begin{array}{l}1.2 \\
0.5\end{array}$ & $\begin{array}{l}0.8 \\
0.4\end{array}$ & $\begin{array}{l}0.6 \\
0.1\end{array}$ \\
\hline
\end{tabular}

a Quantal linear model: $P[$ response $]=$ background $+[1$ - background $] \times$ [1 - EXP (- slope $\times$ dose)].

b $\mathrm{BMD}_{10}$ is the cadmium excretion level in urine which can be expected to yield an excess prevalence of abnormal levels of the substance used as an indication for renal dysfunction of 0.10 .

c $\mathrm{BMDL}_{10}$ is the lower $95 \%$ confidence limit of $\mathrm{BMD}_{10}$.

${ }^{d} \mathrm{BMD}_{5}$ is the cadmium excretion level in urine which can be expected to yield an excess prevalence of abnormal levels of the substance used as an indication for renal dysfunction of 0.05 .

e $\mathrm{BMDL}_{5}$ is the lower $95 \%$ confidence limit of $\mathrm{BMD}_{5}$.

${ }^{\dagger}$ A log-logistic model was used. The intercept value is -2.212 . $\{\log -$ logistic model: P[response $]=$ background $+[1$ - background $] /[1+$ EXP (- intercept - slope $\times \log ($ dose $)]$ \}. for the men and $3.6 \mu \mathrm{g} / \mathrm{g}$ cr for the women when corrected for creatinine and $1.6 \mu \mathrm{g} /$ day for the men and $4.7 \mu \mathrm{g} /$ day for the women for the 24 -hour excretion. The $\mathrm{BMDL}_{10}$ of the urinary cadmium concentration for $\beta_{2}-\mathrm{MG}$ was $0.7 \mu \mathrm{g} / \mathrm{g}$ cr for the men and $1.3 \mu \mathrm{g} / \mathrm{g}$ cr for the women when corrected for creatinine and $1.1 \mu \mathrm{g} /$ day for the men and $1.6 \mu \mathrm{g} / \mathrm{day}$ for the women for the 24 hour excretion. The $\mathrm{BMDL}_{10}$ of the urinary cadmium concentration for NAG was $0.6 \mu \mathrm{g} / \mathrm{g}$ cr for the men and $1.2 \mu \mathrm{g} / \mathrm{g}$ cr for the women when corrected for creatinine and $0.8 \mu \mathrm{g} /$ day for the men and $0.5 \mu \mathrm{g} /$ day for the women for the 24-hour excretion.

\section{Discussion}

It is well known that cadmium contamination from an upstream mine in the Jinzu River basin of the Toyama Prefecture led to the development of Itai-itai disease, representing the most severe stage of chronic cadmium intoxication in many of the inhabitants of this region since World War II (1). In this study 24-hour urine samples were collected from the Kurobe region of the Toyama Prefecture. Because another water system (non-Jin$\mathrm{zu}$ River water system) has been used in this region, this area is thought to be an area without any known environmental cadmium pollution. The geometric means of the cadmium concentrations in urine were $1.9 \mu \mathrm{g} / \mathrm{g} \mathrm{cr}$ for the men and $4.3 \mu \mathrm{g} / \mathrm{g}$ cr for the women. Although the values in the Toyama Prefecture were higher than the values in the Shiga and Wakayama prefectures, the values were at almost the same levels as those of the previous study in the Ishikawa Prefecture (ie, $2.1 \mu \mathrm{g} / \mathrm{g}$ cr for men and $4.0 \mu \mathrm{g} / \mathrm{g}$ cr for women) (14).

In studies of health effects caused by exposure to cadmium, the urinary cadmium concentration is often used as an indicator of internal dose. Reports on cadmium levels of workers and animals have shown a close relationship between urinary cadmium excretion and the total body burden of cadmium (23-27). We found that urinary cadmium increased with the increasing lifetime cadmium intake among inhabitants of the general environment (10). Therefore, we consider urinary cadmium to be useful as an indicator of cadmium exposure in the general environment. Shimbo and his colleagues collected spot urine and peripheral blood samples, together with 24-hour duplicates of food intake from 607 nonsmoking adult women in 30 general survey sites in seven administrative regions throughout Japan between 1991 and 1998 (28). The cadmium concentration in urine, blood, and food duplicates were analyzed. The researchers found that the blood cadmium level correlated closely with the urinary cadmium level, and both the blood cadmium and urinary cadmium levels correlated 
with the cadmium content of food on an individual basis $(\mathrm{N}=607)$, on a survey site basis $(\mathrm{N}=30)$, and on a regional basis $(\mathrm{N}=7)$. They concluded that the urinary cadmium level can be employed as a biomarker of environmental cadmium exposure; that is, also for inhabitants, the urinary cadmium concentration is useful as an indicator of cadmium exposure.

In our previous studies, we used total protein, $\beta_{2}-\mathrm{MG}$, and NAG in urine as indicators of renal dysfunction (13-15). The urinary concentration of total protein is considered to reflect glomerular and tubular dysfunctions as a result of cadmium exposure, and urinary $\beta_{2}-\mathrm{MG}$ and NAG are considered to reflect renal tubular dysfunction. Taylor and his co-workers also showed that these three parameters were useful as indicators of renal dysfunction in an investigation of renal effects caused by exposure to cadmium (29). Thus we used these three parameters as indicators of renal dysfunction in this study.

Our previous studies, which investigated the renal effects of cadmium exposure in areas without any known cadmium pollution, showed a dose-response relationship between cadmium concentration in blood or urine as an indicator of internal dose and indicators of renal dysfunction (total protein, $\beta_{2}-\mathrm{MG}$, and NAG in urine) and suggested the existence of renal effects (13$15)$.

Buchet et al carried out a similar study in Belgium to assess whether environmental exposure to cadmium is associated with renal dysfunction (30). They collected 1699 urine samples from four areas of Belgium with varying degrees of cadmium pollution and found significant associations between cadmium excretion and five variables [urinary excretion of retinol binding protein (RBP), NAG, $\beta_{2}-\mathrm{MG}$, amino acids, and calcium] in agreement with our results.

Ikeda and his co-workers, on the other hand, reported that there was no apparent association between cadmium exposure and an elevation in the urinary low-molecular-weight protein levels of Japanese women living in areas without any known environmental heavy metal pollution (16). The blood and urine samples collected from 607 women were the same samples that had been used in Shimbo's study. The external cadmium dose was evaluated in terms of daily cadmium intake via food, whereas the cadmium levels in blood and urine were taken as internal dose indicators. In contrast to our results, they concluded that they could not find significant dose-dependent changes in the indicators of renal dysfunction $\left[\beta_{2}-\mathrm{MG}, \mathrm{RBP}\right.$, and $\alpha_{1}$-microglobulin $\left.\left(\alpha_{1}-\mathrm{MG}\right)\right]$ using a simple regression analysis or a multiple regression analysis. However, when their multiple linear regression analysis for all 607 participants is examined in detail, the partial regression with the coefficients of the blood cadmium and urinary cadmium concentrations as explanatory variables and the $\alpha_{1}$-MG, $\beta_{2}-\mathrm{MG}$, and RBP as the criterion variables (corrected creatinine) were all significant except for the blood cadmium concentration with respect to RBP. In order to suppress the influence of age to a minimum, the multiple linear regression analysis was carried out for the 367 inhabitants between the ages of 41 and 60 years; it yielded the same results. Furthermore, the use of an analysis of variance in dividing the results into three groups according to the cadmium content of food showed that the blood cadmium and urinary cadmium levels increased as the cadmium content of food increased, and the concentrations of $\alpha_{1}-\mathrm{MG}$ and $\beta_{2}-\mathrm{MG}$ as indicators of renal dysfunction also increased. They concluded that a clear dose-response relationship could not be obtained because, when three kinds of cut-off values (low, middle, and high) for low-molecular-weight protein were used, the prevalences of $\alpha_{1}-\mathrm{MG}$-uria and $\beta_{2}$-MG-uria increased as the cadmium content of food, blood, and urine increased only when the same low cutoff value was used as in our previous studies (13-15), but not when the middle and high cut-off values were used. However, it may not have been appropriate to use the middle or high cut-off value in studying the existence of renal dysfunction with respect to the general environment because only a few people were positive for $\beta_{2}-\mathrm{MG}$ ( 2 and 4 participants of the 367 participants when divided according to the middle and high cut-off values, respectively). However, when the 95\% upper limits of the controls were used to examine the doseresponse relationship among the controls, the number of participants with positive findings was small. In our study, the number of male and female participants with values over the $95 \%$ upper limit was 20 and 21, respectively, and it was difficult to find a dose-response relationship. Thus we used the $84 \%$ upper limit values (65 male and 67 female participants) when we investigated the dose-response relationship in our study.

Thus, although the conclusion of Ikeda and his colleagues differed from ours with respect to the influence of cadmium exposure in the environment, it is meaningful that the significant relationship between cadmium in urine or blood and indicators of renal dysfunction was found either in our previous studies (13-15) or in the study of Ikeda et al (16). It should be mentioned that the urinary cadmium levels were similar in these studies since the studies were carried out on inhabitants living in the general environment. There is an opinion in Japan that the significant relationship between cadmium in urine and indicators of renal dysfunction is found because the excretory mechanism for both is the same or similar. In other words, although there seems to be a dose-response relationship, it does not reflect renal dysfunction by cadmium exposure; that is to say, it is only to observe the phenomenon that those 
who excrete a lot of cadmium are excreting many indicators of renal dysfunction. However, it should be emphasized that there was also a dose-response relationship between cadmium in blood, which is independent of the excretory mechanism, and indicators of renal dysfunction (15).

Buchet et al reported that the critical concentration level of cadmium in urine was estimated to be $2-4 \mu \mathrm{g} /$ day with a $10 \%$ prevalence of values being abnormal, using $\beta_{2}-\mathrm{MG}$ and NAG as indicators of renal dysfunction in the aforementioned study (30). In our study, the $\mathrm{BMDL}_{10}$ of $\beta_{2}$-MG was $1.1 \mu \mathrm{g} /$ day for the men and 1.6 $\mu \mathrm{g} / \mathrm{day}$ for the women; in the case of using the corrected creatinine value, it was $0.7 \mu \mathrm{g} / \mathrm{g}$ cr for the men and $1.3 \mu \mathrm{g} / \mathrm{g}$ cr for the women. The quantal linear model has generally been used to calculate BMD, and this model gave a good fit with the present data. Therefore, the quantal linear model was used to estimate the threshold level of urinary cadmium in this study. Hayano and his colleagues reported that the critical cadmium concentration that corresponded to the prevalence rate of $\beta_{2-}$ MG-uria for the controls in the obtained logistic regression analysis was $1.6-3.0 \mu \mathrm{g} / \mathrm{g}$ cr for the men and 2.3$4.6 \mu \mathrm{g} / \mathrm{g} \mathrm{cr}$ for the women when $\beta_{2}-\mathrm{MG}$ was used as an indicator of renal dysfunction for the inhabitants of the cadmium-polluted Kakehashi River basin (the cut-off value was $1000 \mu \mathrm{g} / \mathrm{g} \mathrm{cr}$ ) (11). On the other hand, Järup and his colleagues reported that a dose-response relationship was found between urinary cadmium and $\alpha_{1}$ MG among 1021 Swedes exposed to cadmium either occupationally or environmentally, with the use of the upper $95 \%$ limit as a cut-off value, and the odds ratio was 6.0 (31). Furthermore, they calculated that an increased tubular proteinuria of $10 \%$ in a 53 -year-old participant (the mean age of the reference group) occurs at a urinary cadmium concentration of $1 \mu \mathrm{g} / \mathrm{g} \mathrm{cr}$. In our study, using $\beta_{2}-\mathrm{MG}$ as an indicator of renal tubular dysfunction, the threshold level of urinary cadmium for a 53year-old participant was calculated to be $0.5 \mu \mathrm{g} / \mathrm{g}$ cr for men and $1.6 \mu \mathrm{g} / \mathrm{g}$ cr for women when the same procedure as Järup's was used. It is thought that further investigation is necessary to determine whether it is appropriate to adopt the BMDL value in the calculation of the critical concentration level of cadmium exposure in the environment. In a summary of our aforementioned studies, the threshold level of urinary cadmium in Japan was inferred to be almost the same as in Belgium and Sweden.

The significance of the slight kidney effect observed in our study is not yet clear. However, it should be pointed out that even a slightly increased urinary excretion of $\beta_{2}$-MG $(300 \mu \mathrm{g} / \mathrm{g} \mathrm{cr}<)$ is associated with increased mortality (32). The cut-off values $233 \mu \mathrm{g} / \mathrm{g}$ cr for men and $274 \mu \mathrm{g} / \mathrm{g}$ cr for women were used for $\beta_{2}-\mathrm{MG}$ in the analyses in our study; these values do not differ much from the $300 \mu \mathrm{g} / \mathrm{g}$ used in the previous study. From this point of view, the existence of a dose-response relationship for cadmium exposure in the general environment is thought to be important and indicates an urgent need for another study evaluating the significance of this relationship, especially with respect to mortality in the general population.

\section{References}

1. Nogawa K. Itai-Itai disease and follow-up studies. In: Nriagu JO, editor. Cadmium in the environment, part 2: health effets. New York, Chichester, Brisbane, Toronto, Singapore: Wiley Interscience Publication; 1981. p 1-37.

2. Nomiyama K. The chronic toxicity of cadmium: influence of environment and other variables. In Foulkes EC, editor. Handbook of environmental pharmacology. Berlin, Heideberg, New York, Tokyo: Spring-Verlag; 1986. p 101-33.

3. Nakashima K, Kobayashi E, Nogawa K, Kido T, Honda R. Concentration of cadmium in rice and urinary indicators of renal dysfunction. Occup Environ Med 1997;54:750-5.

4. Osawa T, Kobayashi E, Okubo Y, Suwazono Y, Kido T, Nogawa K. A retrospective study on relation between renal dysfunction and cadmium concentration in rice in individual hamlets in the Jinzu River basin, Toyama Prefecture. Environ Res 2001;86:51-9.

5. Watanabe Y, Kobayashi E, Okubo Y, Suwazono Y, Kido T, Nogawa K. Relationship between cadmium concentration in rice and renal dysfunction in individual subjects of the Jinzu river basin determined using a logistic regression analysis. Toxicology 2002;172:93-101.

6. Kido T, Nogawa K. Dose-response relationship between total cadmium intake and $\beta 2$-microglobulinuria using logistic regression analysis Toxicol Lett 1993;69:113-20.

7. Kido T, Shaikh ZA, Kito H, Honda R, Nogawa K. Doseresponse relationship between total cadmium intake and metallothioneinuria using logistic regression analysis. Toxicology 1993;80:207-15.

8. Hochi Y, Kido T, Nogawa K, Kito H, Shaikh ZA. Doseresponse relationship between total cadmium intake and prevalence of renal dysfunction using general linear models. J Appl Toxicol 1995;15:109-16.

9. Kobayashi E, Okubo Y, Suwazono Y, Kido T, Nogawa K. Dose-response relationship between total cadmium intake calculated from the cadmium concentration in rice collected from each household of farmer and renal dysfunction in inhabitants of the Jinzu River basin, Japan. J Appli Toxicol 2002;22:431-6.

10. Kido T, Ohmichi M, Nogawa K, Honda R, Tsuritani I, Ishizaki M, et al. Significance of urinary cadmium concentration in a Japanese population environmentally exposed to cadmium. Arch Environ Health 1992;47:196-202.

11. Hayano M, Nogawa K, Kido T, Kobayashi E, Honda R, Tsuritani I. Dose-response relationship between urinary cadmium concentration and $\beta 2$-microglobulinuria using logistic regression analysis. Arch Environ Health 1996;51:162-7.

12. Chiyoda N, Kobayashi E, Okubo Y, Suwazono Y, Kido T, Nogawa K. Allowable level of lifetime cadmium intake calculated from the individuals in the Jinzu River basin of Japan. Biol Trace Elem Res 2003;96:9-20. 
13. Yamanaka O, Kobayashi E, Nogawa K, Suwazono Y, Sakurada I, Kido T. Association between renal effects and cadmium exposure in cadmium-nonpolluted area in Japan. Environ Res 1998;77:1-8.

14. Yin Kyi Oo, Kobayashi E, Nogawa K, Okubo Y, Suwazono Y, Kido T, et al. Renal effects of cadmium intake of a Japanese general population in two areas unpolluted by cadmium. Arch Environ Health 2000;55:98-103.

15. Suwazono Y, Kobayashi E, Nogawa K, Okubo Y, Kido T, Nakagawa H. Renal effects of cadmium exposure in cadmium nonpolluted areas in Japan. Environ Res 2000;84:44-55.

16. Ikeda M, Zhang Z-W, Moon C-S, Shimbo S, Watanabe T, Nakatsuka H, et al. Possible effects of environmental cadmium exposure on kidney function in the Japanese general population. Int Arch Occup Environ Health 2000;73:15-25.

17. Stamler J, Elliott P, Dennis B, Dyer AR, Kesteloot H, Liu K, et al. INTERMAP: background, aims, design, methods, and descriptive statistics (non-dietary). J Human Hypertens 2003;17:591-608.

18. Ueshima H, Okayama A, Saitoh S, Nakagawa H, Rodriguez $\mathrm{B}$, Sakata K, et al. Differences in cardiovascular disease risk factors between Japanese in Japan and Japanese-Americans in Hawaii: the INTERLIPID study. J Human Hypertens 2003; 17:631-9.

19. Kido T, Tsuritani I, Honda R, Ishizaki M, Yamada Y, Nogawa $\mathrm{K}$. A direct determination of urinary cadmium by graphitefurnace atomic absorptionspectrometry using the zeeman effect [in Japanese]. J Kanazawa Med Univ 1984;9:70-5.

20. Bonsnes RW, Taussky HH. On the colorimetric determination of creatinine by the Jaffe reaction. J Biol Chem 1945;158:58191.

21. Crump KS. A new method for determining allowable daily intakes. Fundam Appl Toxicol 1984;4:854-71.

22. Crump KS. Calculation of benchmark dose from continuous date. Risk Anal 1995;15:79-89.

23. Mueller PW, Smith SJ, Steinberg KK, Thun MJ. Chronic renal tubular effects in relation to urine cadmium levels. Nephron 1989; 52:45-54.

24. Jarup L, Persson B, Elinder CG. Blood cadmium as an indica- tor of dose in a long-term follow-up of workers previously exposed to cadmium. Scand J Work Environ Health 1997; 23:31-6.

25. Jakubowski M, Trzcinka-Ochocka M, Halatek T, Razniewska G, Szymczak W. Integrated indexes of occupational exposure as predictors of kidney dysfunction. Int J Occup Med Environ Health 2002; 15:393-9.

26. Aoyagi T, Hayakawa K, Miyaji K, Ishikawa H, Hata M. Cadmium nephtotoxicity and evacuation from the body in a rat modeled subchronic intoxication. Int J Urol 2003; 10:3328 .

27. Brzoska MM, Kaminski M, Supernak-Bobko D, Zwierz K, Moniuszko-Jakoniuk J. Changes in the structure and function of the kidney of rats chronically exposed to cadmium, 1: biochemical and histopathological studies. Arch Toxicol 2003; 77:344-52.

28. Shimbo S, Zhang Z-W, Moon C-S, Watanabe T, Nakatsuka $\mathrm{H}$, Matsuda-Inoguchi N, et al. Correlation between urine and blood concentrations, and dietary intake of cadmium and lead among women in the general population of Japan. Int Arch Occup Environ Health 2000;73:163-70.

29. Taylor SA, Chivers ID, Price RG, Tomas MA, Milligan P, Francini I, et al. The assessment of biomarkers to detect nephrotoxicity using an integrated database. Environ Res 1997;75:23-33.

30. Buchet JP, Lauwerys RR, Roles H, Bernard A, Claeys F, Ducoffer G, et al. Renal effects of cadmium body burden of the general population. Lancet 1990;336:699-702.

31. Järup L, Hellström L, Alfvén T, Calsson MD, Grubb A, Persson B, et al. Low level exposure to cadmium and early kidney damage: the OSCAR study. Occup Environ Med 2000;57:668-72.

32. Nakagawa H, Nishijo M, Morikawa Y, Tabata M, Senma M, Kitagawa $\mathrm{Y}$, et al. Urinary $\beta 2$-microglobulin concentration and mortality in a cadmium-polluted area. Arch Environ Health 1993,48:428-35.

Received for publication: 20 January 2004 